\title{
OPTIMIZACIÓN CON INCERTIDUMBRE EN ECOSIMPRO
}

\author{
Tania Rodríguez Blanco \\ tania.rodriguez@autom.uva.es \\ Daniel Sarabia Ortiz \\ dsarabia@ubu.es \\ César de Prada Moraga \\ prada@autom.uva.es
}

\begin{abstract}
Resumen
La operación óptima del proceso usando "real time optimization (RTO)" no está siempre garantizada debido a la presencia de incertidumbre en los modelos que son utilizados para la toma de decisiones y debido a las diferencias que existen entre las capas de la estructura de control que operan en diferente escalas de tiempo y usan distintas clases de modelos. La metodología de adaptación por modificadores, más conocida como “Modifier-adaptation (MA)", sirve para alcanzar la optimalidad a pesar de la presencia de incertidumbre, utilizando para ello medidas de la planta.
\end{abstract}

Normalmente, los modificadores son actualizados usando información del estado estacionario para calcular los gradientes del proceso, lo que implica una lenta convergencia hacia el óptimo real. Para acelerar la convergencia del método, en este artículo se estiman los gradientes a partir de información del transitorio usando el método de estimación de mínimos cuadrados recursivo. En este artículo se presenta la implementación de esta metodología sobre la simulación del reactor Otto-Williams.

Palabras Clave: Optimización en tiempo real, incertidumbre, modifier-adaptation.

\section{INTRODUCCIÓN}

La gestión de sistemas de gran escala, como pueden ser muchos procesos de la industria petroquímica, consiste en tomar decisiones que satisfagan especificaciones de proceso y restricciones en muchas de las variables. Además, estas decisiones deben ser óptimas desde un punto de vista de eficiencia, económico y medio ambiental. Éste problema requiere el uso de modelos de gran dimensión y métodos de optimización.
RTO consiste en una capa de optimización que está presente sobre la capa de control y en la que se toman decisiones en una escala de tiempo de horas a días considerando objetivos económicos explícitamente. El punto de operación óptimo obtenido por dicha capa es pasado a las capas de control que pueden incluir control básico o control predictivo multivariable (MPC). Sin embargo, la operación óptima no está garantizada ya que los modelos del proceso real nunca son exactos, de modo que el óptimo del proceso puede no ser el mismo que el óptimo calculado a partir del modelo. Además las diferentes capas de control usan diferentes tipos de modelos, por ejemplo, RTO está basada en modelos de primeros principios no lineales que describen el comportamiento estacionario de la planta, mientras que el MPC se basa en modelos dinámicos lineales. Esto produce incoherencias entre las distintas capas que pueden afectar al resultado final.

Para tratar con la incertidumbre ya mencionada y conducir el proceso a su óptimo real se han desarrollado varias técnicas. El primer método surgió en los años 70 como un algoritmo de dos etapas iterativo que incluye un paso de estimación de parámetros (para actualizar los parámetros inciertos del modelo) seguido por una etapa de optimización económica que es resuelta para obtener las nuevas variables de decisión [1]. Pero esta formulación funciona correctamente solo si hay poca diferencia estructural entre el modelo y el proceso real y si las condiciones de operación cambiantes aportan suficiente excitación para estimar los parámetros inciertos correctamente [2].

Posteriormente, un nuevo enfoque fue desarrollado por Roberts quien incorporó información referente a los gradientes de la planta añadiendo un nuevo modificador que resulta de la diferencia entre el gradiente de la función de coste real y el del modelo [3]. Éste método se llamó “integrated system optimization and parameter estimation" (ISOPE). En 2002, Tatjewski demostró que la convergencia hacia el punto óptimo no depende de la estimación de 
parámetros sino de la igualdad entre las salidas del proceso y del modelo medidas en cada iteración del RTO [4]. Por esta razón, introdujo un nuevo modificador que tiene en cuenta la diferencia entre dichas salidas. Nuevos modificadores también fueron añadidos por Gao y Engell para las restricciones de proceso [5]. La formulación general de esta metodología se muestra en la Figura 1.

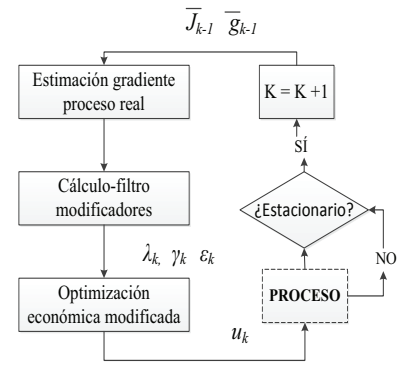

Figura 1: Formulación general metodología de adaptación por modificadores

A partir de estas ideas han surgido varios métodos, la mayoría de los cuales requieren el cálculo de gradientes experimentales lo cual supone una difícil tarea.

Uno de estos métodos es el conocido como Dual Modifier Adaptation (DMA) [6] que estima gradientes experimentales a partir de puntos de operación pasados, generados por RTO previas, usando la definición de derivada direccional. Para asegurar que los gradientes son obtenidos con exactitud, una nueva restricción es añadida al problema de optimización $\left(\delta\left(\mathrm{S}_{\mathrm{K}}\right) \geq \delta^{\mathrm{L}}\right)$ siendo $\mathrm{S}_{\mathrm{K}}$ la matriz formada por los vectores de diferencias de las variables de decisión con respecto a $n_{\mathrm{u}}$ períodos anteriores, $\delta$ la inversa del número de condición de la matriz $\mathrm{S}_{\mathrm{K}}$ y $\delta^{\mathrm{L}}$ el parámetro que indica el mínimo grado de excitación del proceso. Ésta restricción representa la característica dual del método: mientras el resto de la optimización intenta converger al óptimo del modelo modificado (primal objective), la restricción dual garantiza que en la siguiente iteración del RTO el sistema tendrá suficiente energía para estimar el gradiente del proceso adecuadamente (objetivo dual).

El cálculo directo de los gradientes experimentales puede ser evitado usando una formulación llamada Nested- Modifier Adaptation (NMA) [7]. Éste método usa una arquitectura de optimización anidada con un algoritmo de optimización no basado en gradientes, como el método Nelder- Mead, para actualizar los modificadores, iterando con ellos sobre el problema de optimización modificado hasta que se alcanza el óptimo del proceso. De este modo, la estimación del gradiente del proceso real es sustituida por otro método que tiene en cuenta la minimización de la función de coste medida directamente de la planta.

Una de las principales desventajas de MA es la necesidad de esperar a que el proceso alcance el estado estacionario para actualizar el valor de los modificadores. En muchas aplicaciones reales, como por ejemplo las columnas de destilación, este estado puede alcanzarse después de varias horas por lo que la convergencia de MA sería muy lenta y el óptimo real se alcanzaría después de días de operación. Durante este largo período de tiempo las condiciones de operación o la incertidumbre entre el proceso y el modelo podrían cambiar de modo que el óptimo nunca se alcanzaría. Este problema hace que la implementación de esta técnica en procesos reales no sea práctica en estos casos.

Para solucionar este problema se ha implementado la metodología MA usando medidas del transitorio para estimar el valor de los gradientes del proceso permitiendo así una mayor velocidad de convergencia hacia el óptimo real. El buen funcionamiento de este método es presentado a través de la implementación sobre la simulación del reactor Otto- Williams.

La estructura a seguir será la siguiente, primero, la descripción de la nueva metodología de MA basada en medidas del transitorio. A continuación, se describe el caso de estudio sobre el que se implementarán las técnicas descritas. En la sección 4 se muestran los resultados obtenidos después de la implementación de las citadas técnicas y por último las principales conclusiones extraídas de este trabajo.

\section{ADAPTACIÓN DE MODIFICADORES USANDO INFORMACIÓN DEL TRANSITORIO.}

Como se muestra en la Figura 1, la metodología MA requiere la estimación del gradiente del proceso para calcular los modificadores. En algunos casos, podría no ser factible determinar el valor preciso de este gradiente. Por ello, se ha decidido deducir este "parámetro" mediante observación de la respuesta del sistema utilizando una técnica de estimación de parámetros como el método de mínimos cuadrados recursivo.

El enfoque considerado aplica la metodología MA usando medidas del transitorio para estimar los gradientes de la planta y consecuentemente el valor de los modificadores, permitiendo la convergencia al óptimo más rápido que el método tradicional basado en información del estacionario. 
Para implantar esta metodología, se toman medidas en cada instante de optimización (durante el transitorio) para estimar los modificadores empleando para ello técnicas de estimación adaptativas cuya solución provea las nuevas entradas $u$ que deben aplicarse a la planta hasta la siguiente optimización.

\subsection{ESTIMACIÓN DEL GRADIENTE DE LA PLANTA}

Como se ha dicho anteriormente, una técnica de estimación de parámetros será utilizada para estimar los gradientes del proceso y además los modificadores del problema RTO modificado. El ingrediente esencial del problema de estimación de parámetros es elegir la clase de modelo. En este caso, la dinámica de la función de coste ha sido parametrizada como se muestra en (1) considerando un modelo DARMA de primer orden que relaciona la variación de la función de coste del proceso $\Delta J_{k}$ con la variación de las entradas $\Delta u_{k}$ (variables de decisión del problema RTO):

$$
\Delta J_{k}=\theta_{k}{ }^{T} \Delta u_{k}=\phi_{k}^{T} \theta_{k}
$$

Siendo el estimador de la variación de la función de coste:

$$
\Delta \hat{J}_{k}=\hat{\theta}_{k}{ }^{T} \Delta u_{k}=\phi_{k}{ }^{T} \hat{\theta}_{k}
$$

Donde $\hat{\theta}_{k}$ es el vector de parámetros estimados que contiene los gradientes del proceso necesarios para calcular los modificadores, es decir, los gradientes de la función de coste del proceso con respecto a las variables de decisión $u_{k}$.

El gradiente $\theta_{k}$ es estimado empleando el método de mínimos cuadrados recursivo con factor de olvido $\alpha$. El concepto de olvido significa que los datos más antiguos son gradualmente descartados a favor de la información más reciente [12].

Este algoritmo ha sido aplicado por su simplicidad y fácil implementación, además, generalmente tiene una convergencia más rápida que otros métodos como el algoritmo de proyección. Esta técnica también es válida en presencia de ruido blanco que es inherente a todo proceso real.

Se basa en la diferencia entre la entrada actual $u_{k}$ y la aplicada en el período anterior y la diferencia entre el cambio de la función de coste medida $\Delta J_{k} \mathrm{y}$ la predicha $\Delta \hat{J}_{k}[13]$. El método de estimación de parámetros utilizado viene descrito por las siguiente $\mathrm{s}$ ecuaciones, siendo $\phi_{k}=\Delta u_{k}$ :

$$
\begin{gathered}
\Sigma_{0}=\frac{1}{\alpha} I \\
\phi_{k}=\Delta u_{k}=u_{k}-u_{k-1}
\end{gathered}
$$

$$
\begin{gathered}
\Delta \hat{J}_{k}=\phi_{k}{ }^{T} \hat{\theta}_{k} \\
e_{k}=\Delta J_{k}-\Delta \hat{J}_{k} \\
\Sigma_{k+1}^{-1}=\frac{1}{\alpha} \Sigma_{k}^{-1}-\frac{1}{\alpha^{2}} \Sigma_{k}^{-1} \phi_{k}\left(+\frac{1}{\alpha} \phi_{k}{ }^{T} \Sigma_{k}^{-1} \phi_{k}\right)^{-1} \phi_{k}{ }^{T} \Sigma_{k}^{-1} \\
\hat{\theta}_{k+1}=\hat{\theta}_{k}+\frac{1}{\alpha} \Sigma_{k}^{-1} \phi_{k}\left(1+\frac{1}{\alpha} \phi_{k}{ }^{T} \Sigma_{k}^{-1} \phi_{k}\right)^{-1}\left(e_{k}\right)
\end{gathered}
$$

Donde $\Sigma$ es la matriz de covarianza, $e_{k}$ el error de predicción, $u_{k}$ es la solución del RTO modificado para el período de muestreo $k$. Además, $\Delta J_{k}=J_{k}-$ $J_{k-1}$ es la diferencia entre el valor de la función de coste actual y la medida en un período de muestreo anterior.

\section{CASO DE ESTUDIO}

En esta sección el reactor Otto- Williams es considerado como caso de estudio para comparar el rendimiento del nuevo enfoque basado en el transitorio con el método MA tradicional. Se trata de un reactor continuo de tanque agitado que ha sido ampliamente utilizado como caso de estudio para la comparación de diferentes técnicas RTO en presencia de discrepancias proceso- modelo [3], [6], [11], [12].

El sistema considerado es alimentado con dos tipos de materias primas $A$ y $B$, con un flujo igual a $F_{A}$ y $F_{B}$ respectivamente. Dentro del equipo tienen lugar tres reacciones en paralelo dando lugar a 4 nuevos compuestos: $C, G, E$ y $P$, como se muestra en la ecuación (15). Dichos compuestos, además de los reactivos restantes, abandonan el reactor en una única corriente $F_{R}$. $X_{i}$ representa la fracción másica del componente $i$ dentro del reactor y $T_{R}$ la temperatura del reactor.

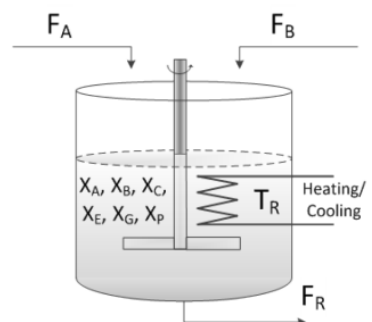

Figura 2: Diagrama del reactor Otto-Williams

\subsection{PROCESO REAL}

El sistema puede describirse usando un modelo de primeros principios que ha sido simulado en el programa de modelado y simulación EcosimPro. Los balances de materia para cada uno de los compuestos se definen como se indica a continuación: 


$$
\begin{aligned}
& A+B \stackrel{k_{1}}{\rightarrow} C, \quad B+C \stackrel{k_{2}}{\rightarrow} P+E, \quad C+P \stackrel{k_{3}}{\rightarrow} G \\
& V_{R} \frac{d X_{A}}{d t}=F_{A}-F_{R} X_{A}-V_{R} r_{1} \\
& V_{R} \frac{d X_{B}}{d t}=F_{B}-F_{R} X_{B}-V_{R} r_{1} \frac{M_{B}}{M_{A}}-V_{R} r_{2} \\
& V_{R} \frac{d X_{C}}{d t}=-F_{R} X_{C}+V_{R} r_{1} \frac{M_{C}}{M_{A}}-V_{R} r_{2} \frac{M_{C}}{M_{B}}-V_{R} r_{3} \\
& V_{R} \frac{d X_{E}}{d t}=-F_{R} X_{E}+V_{R} r_{2} \frac{M_{E}}{M_{B}} \\
& V_{R} \frac{d X_{G}}{d t}=-F_{R} X_{G}+V_{R} r_{3} \frac{M_{G}}{M_{C}} \\
& V_{R} \frac{d X_{P}}{d t}=-F_{R} X_{P}+V_{R} r_{2} \frac{M_{P}}{M_{B}}-V_{R} r_{3} \frac{M_{P}}{M_{C}} \\
& F_{R}=F_{A}+F_{B}
\end{aligned}
$$

Donde $M i$ representa el peso molecular del compuesto $i$, y $r_{j}$ es la velocidad de la reacción $j$ definida con respecto a su reactivo limitante.

Al trabajar con pseudo-compuestos es necesario definir la relación entre sus pesos moleculares. Esto se puede obtener considerando que $\mathcal{M} A=\mathcal{M} B=\mathcal{M} P$. Bajo esta suposición los cocientes entre pesos moleculares que aparecen en las ecuaciones (10-15) pueden obtenerse como:

$$
\frac{M_{B}}{M_{A}}=\frac{M_{P}}{M_{B}}=1, \frac{M_{C}}{M_{A}}=\frac{M_{C}}{M_{B}}=\frac{M_{E}}{M_{B}}=2, \frac{M_{G}}{M_{C}}=1.5, \frac{M_{P}}{M_{C}}=0.5
$$

La velocidad de cada una de las reacciones se calcula según (18-20):

$r_{1}=k_{1} X_{A} X_{B}$

$r_{2}=k_{2} X_{B} X_{C}$

$r_{3}=k_{3} X_{C} X_{P}$

Donde $k_{j}$ es la constante cinética de la reacción $j$ que se obtiene a partir de la ecuación de Arrhenius, siendo $E_{A j}$ la energía de activación.

$k_{j}=k_{j}^{0} e^{\left(\frac{E_{A j}}{T_{R}}\right)}, \quad j=1,2,3$

\subsection{FUNCIÓN DE COSTE DEL PROCESO}

El objetivo es maximizar el beneficio de la operación que se expresa como la diferencia de coste entre el producto obtenido y las materias primas empleadas, suponiendo que entran con una fracción másica igual a 1.

$J=F_{R}\left(X_{P} P_{P}+X_{E} P_{E}\right)-F_{A} C_{A}-F_{B} C_{B}$

El flujo de reactivo A $\left(F_{A}\right)$ se fija en un valor igual a $1.8275 \mathrm{~kg} / \mathrm{s}$. El flujo de reactive $\mathrm{B}\left(F_{B}\right)$ y la temperatura del reactor $\left(T_{R}\right)$ son las variables de decisión del problema de optimización, de modo que $u=\left(F_{B}, T_{R}\right)^{T}$.

\subsection{PUNTO DE OPERACIÓN ÓPTIMO DEL PROCESO}

El punto de operación óptimo de la planta (realidad simulada) se muestra en la Tabla 1. Las fracciones másicas obtenidas en la solución óptima se presentan en la Tabla 2.

Tabla 1: Óptimo del proceso

\begin{tabular}{|c|c|c|}
\hline $\mathbf{F}_{\mathbf{B}}(\mathrm{Kg} / \mathbf{s})$ & $\mathbf{T}_{\mathbf{R}}$ (으) & $\mathbf{J}(\mathbf{\$} / \mathbf{s})$ \\
\hline 4.78 & 89.70 & 191.22 \\
\hline
\end{tabular}

Tabla 2: Fracciones másicas en el óptimo

\begin{tabular}{|c|c|c|c|c|c|}
\hline $\mathbf{X}_{\mathbf{A}}{ }^{*}$ & $\mathbf{X}_{\mathrm{B}}{ }^{*}$ & $\mathbf{X}_{\mathbf{C}}{ }^{*}$ & $\mathbf{X}_{\mathrm{E}}{ }^{*}$ & $\mathbf{X}_{\mathbf{G}}{ }^{*}$ & $\mathbf{X}_{\mathbf{P}}{ }^{*}$ \\
\hline 0.0874 & 0.3896 & 0.0153 & 0.2906 & 0.1075 & 0.1094 \\
\hline
\end{tabular}

\subsection{MODELO ESTACIONARIO CAPA RTO}

El valor de la fracción másica del producto $\mathrm{C}$ es de un orden de magnitud por debajo del resto de los componentes. Por ello, una aproximación del proceso es considerar únicamente las otras 5 especies lo que implica una gran diferencia entre el proceso y el modelo. De este modo, se considera que sólo tienen lugar dos reacciones en paralelo:

$A+2 B \stackrel{\tilde{k}_{1}}{\rightarrow} P+E, \quad A+B+P \stackrel{\tilde{k}_{2}}{\rightarrow} G+E$

Considerando esta fuente de incertidumbre, el modelo en estado estacionario que será usado para la optimización de la capa RTO viene descrito por las siguientes ecuaciones:

$F_{A}-F_{R} X_{A}-V_{R} \tilde{r}_{1}-V_{R} \tilde{r}_{2}=0$

$F_{B}-F_{R} X_{B}-2 V_{R} \tilde{r}_{1}-V_{R} \tilde{r}_{2}=0$

$-F_{R} X_{E}+2 V_{R} \tilde{r}_{1}=0$

$-F_{R} X_{G}+3 V_{R} \tilde{r}_{2}=0$

$-F_{R} X_{P}+V_{R} \tilde{r}_{1}-V_{R} \tilde{r}_{2}=0$

$F_{R}=F_{A}+F_{B}$

$\tilde{r}_{1}=\tilde{k}_{1} X_{A}\left(X_{B}\right)^{2}$

$\tilde{r}_{2}=\tilde{k}_{2} X_{A} X_{B} X_{P}$

$\tilde{k}_{j}=\tilde{k}_{j}^{0} e^{\left(\frac{\widetilde{E}_{A j}}{T_{R}}\right)}, \quad j=1,2$

Donde la tilde representa los parámetros utilizados en el modelo que presenta la incertidumbre.

\subsection{FORMULACIÓN PROBLEMA DE OPTIMIZACIÓN}

En resumen, el problema de optimización de la capa RTO se resumen en encontrar las variables de decisión $\mathrm{F}_{B}$ y $T_{R}$ que maximicen el beneficio de la planta, sujeto a un modelo que solo considera 5 compuestos y dos reacciones químicas lo cual supone 
una alta discrepancia proceso-modelo. El problema de optimización se ha resuelto utilizando un algoritmo de programación cuadrática secuencial (SQP) implementado en la librería SNOPT en EcosimPro.

$\min _{F_{B, T},}-J$

s.t.:

steady - state model with mismatch

$F_{B} \in\left[F_{B}^{L}, F_{B}^{U}\right]$

$T_{R} \in\left[T_{R}^{L}, T_{R}^{U}\right]$

Los valores de los parámetros presentes en las ecuaciones (10-32) se resumen en la Tabla 3.

Tabla 3: Valor de los parámetros del modelo

\begin{tabular}{|c|c|c|c|}
\hline Parámetro & Valor & Parámetro & Valor \\
\hline $\mathrm{F}_{A}$ & 1.8725 & $\widetilde{E}_{A 1}$ & -8077.6 \\
\hline$V_{R}$ & 2105 & $\widetilde{E}_{A 2}$ & -12438.5 \\
\hline$k_{1}{ }^{0}$ & $1.6599 \times 10^{6}$ & $F_{B}^{L}$ & 3 \\
\hline$k_{2}{ }^{0}$ & $7.2177 \times 10^{8}$ & $F_{B}^{U}$ & 6 \\
\hline$k_{3}{ }^{0}$ & $2.6745 \times 10^{12}$ & $T_{R}^{L}$ & 70 \\
\hline$E_{A 1}$ & -6666.7 & $T_{R}^{U}$ & 100 \\
\hline$E_{A 2}$ & -8333.3 & $\mathrm{P}_{\mathrm{P}}$ & 1143.38 \\
\hline$E A 3$ & -11111 & $\mathrm{P}_{\mathrm{E}}$ & 25.92 \\
\hline$\tilde{k}_{1}^{0}$ & $2.611 \times 10^{13}$ & $C_{A}$ & 76.23 \\
\hline$\tilde{k}_{2}^{0}$ & $1.655 \times 10^{8}$ & $C_{B}$ & 114.34 \\
\hline
\end{tabular}

El problema de optimización modificado que se resuelve en cada iteración del RTO se describe como:

$\min _{F_{B, T_{R}}}-J+\lambda_{1}\left(F_{B, k}-F_{B, k-1}\right)+\lambda_{2}\left(T_{R, k}-T_{R, k-1}\right)$

s.t.:

steady - state model with mismatch

$F_{B} \in\left[F_{B}^{L}, F_{B}^{U}\right]$

$T_{R} \in\left[T_{R}^{L}, T_{R}^{U}\right]$

Donde los modificadores se calculan como:

$\lambda_{1}=\frac{\partial J_{p}}{\partial F_{B}}-\frac{\partial J}{\partial F_{B}}$

$\lambda_{2}=\frac{\partial J_{p}}{\partial T_{R}}-\frac{\partial J}{\partial T_{R}}$

Donde $J_{p}$ es el valor de la función de coste medida directamente del proceso.

\section{RESULTADOS}

\subsection{MA a partir de información estacionaria}

El método DMA ha sido implementado sobre el reactor Otto-Williams con un valor de a igual a 0.01 $(\delta \geq a)$, restricción dual que asegura que en la siguiente iteración del RTO el sistema tendrá suficiente excitación para estimar el gradiente con exactitud. El problema RTO es resuelto cada hora para asegurar que el proceso alcanza el estado estacionario en cada itereación. Las Figuras 3 y 4 muestran la evolución de las variables de decisión del RTO y la Figura 5 presenta cómo varía la función de coste del proceso:

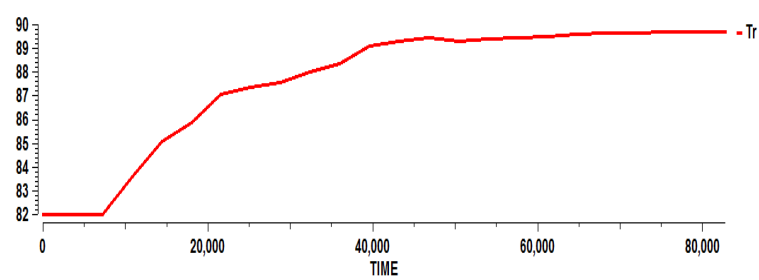

Figura 3. Evolución de la variable de decisión $\mathrm{F}_{\mathrm{B}}$

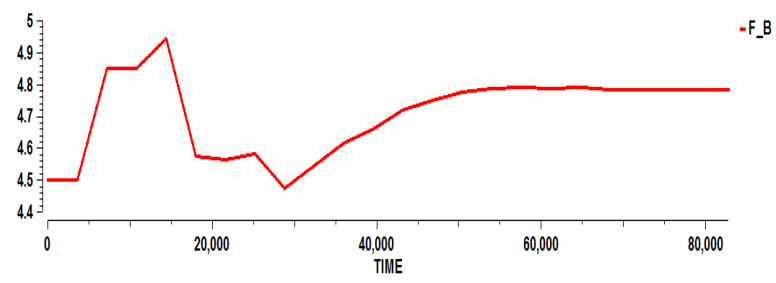

Figura 4. Evolución de la variable de decisión $T_{R}$

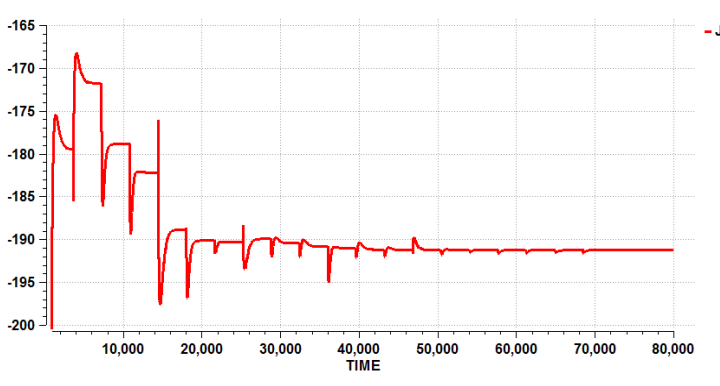

Figura 5. Evolución de la función de coste del proceso $J_{P}$

En vista de los resultados, aplicando MA estático el punto óptimo de operación se alcanza después de 82800 s ( 23 horas). Esto supone esperar 23 estados estacionarios, 21 soluciones del RTO más $n_{u}$ estados iniciales necesarios para estimar los gradientes, siendo $n_{u}$ el número de variables de decisión. Durante este largo período de tiempo las condiciones de operación y las discrepancias entre modelo y proceso podrían cambiar resultando en una pérdida de optimalidad ya que el método MA no lograría converger al óptimo del proceso.

\subsection{MA a partir de información del transitorio}

MA basado en medidas del transitorio ha sido implementado con un período de muestreo del RTO de 600 segundos lo que supone un sexto del tiempo 
de estabilización del proceso. El factor de olvido considerado para el método de estimación del gradiente del proceso ha sido 0.50. Las Figuras 6 y 7 muestran la evolución de las variables de decisión del problema RTO y la Figura 8 la evolución de la función de coste del proceso:

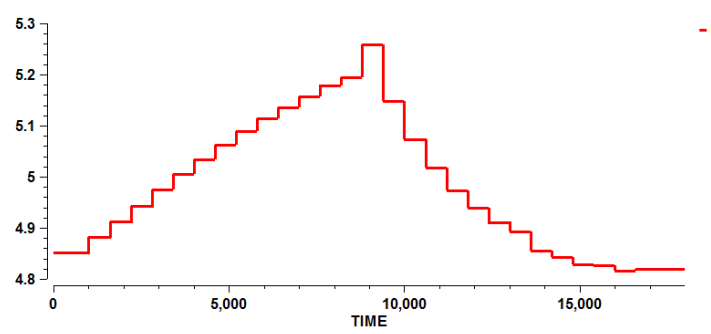

Figura 5. Evolución de la variable de decisión $\mathrm{F}_{\mathrm{B}}$

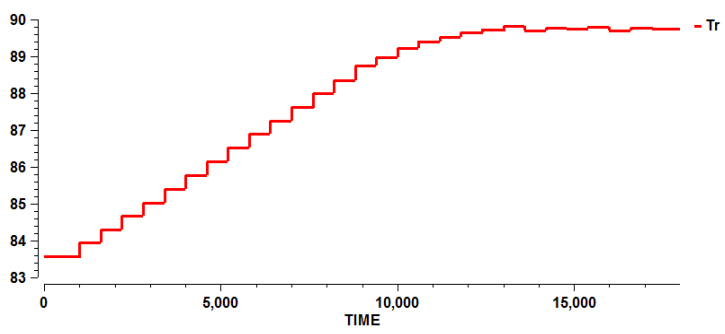

Figura 6. Evolución de la variable de decisión $T_{R}$

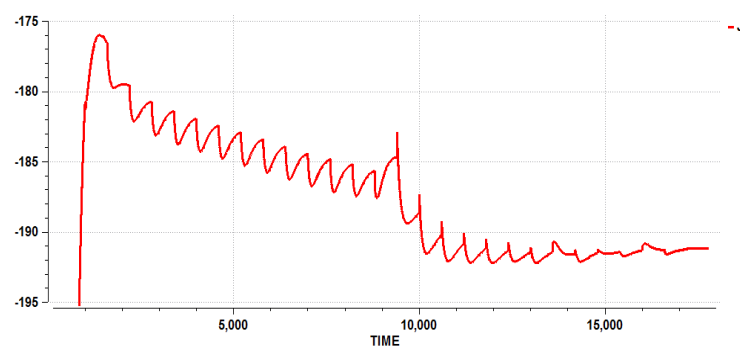

Figura 7. Evolución de la función de coste del proceso $J_{P}$

Aplicando el nuevo enfoque, actualizando los modificadores durante el transitorio, el punto de operación óptimo se alcanza después de 16800 segundos, aproximadamente 4.5 horas, lo que supone una reducción de tiempo considerable con respecto al método MA estático. Este tiempo supone 28 soluciones del RTO ejecutadas durante el transitorio.

\section{CONCLUSIONES}

Aplicando la metodología MA a partir de información del estacionario es posible alcanzar el óptimo del proceso, sin embargo puede que este punto de operación se alcance después de un largo período de tiempo. Por ello se han utilizado medidas en el transitorio para el cálculo de los modificadores, acelerando la convergencia del método.
La técnica propuesta se basa en estimar los gradientes del proceso necesarios para el cálculo de los modificadores utilizando la técnica de estimación de mínimos cuadrados recursiva con factor de olvido. A la vista de los resultados se observa que el óptimo del proceso se alcanza reduciendo en 5 veces el tiempo requerido aplicando el método tradicional.

\section{Agradecimientos}

Los autores desean agradecer al MINECO Español por su apoyo financiero para llevar a cabo este trabajo dentro del proyecto DPI2015-70975-P, además de la beca FPI BES-2013-062737 otorgada al primer autor.

\section{Referencias}

[1] C.Y. Chen, B. Joseph. "On-line optimization using a two-phase approach: An application study". Industrial \& Engineering Chemistry Research, 26, 1987, pp.1924-1930.

[2] W.S. Yip, T.E. Marlin. "The effect of model fidelity on real-time optimization performance". Computers \& Chemical Engineering, 2004; 28, pp.267-280.

[3] P.D. Roberts. "An Algorithm for Steady-State System Optimization and ParameterEstimation". International Journal of Systems Science, 1979; 10, pp.719-734.

[4] P. Tatjewski. "Iterative Optimizing Set-Point Control- The Basic Principle Redesigned". In 15th Triennial World Congress, IFAC. Barcelona, Spain, 2002.

[5] W. Gao, S. Engell. "Iterative set-point optimization of batch chromatography". Computers \& Chemical Engineering, 29, 2005; pp.1401-1409.

[6] A. Marchetti, B. Chachuat, D. Bonvin. "A dual modifier-adaptation approach for real-time optimization". Journal of Process Control, 2010; 20, pp.1027-1037.

[7] D. Navia, G. Gutiérrez, C. de Prada. "Nested Modifier-Adaptation for RTO in the Otto Williams Reactor". In 10th International Symposium on Dynamics and Control of Process Systems, IFAC. Mumbai, India, 2013.

[8] G.C. Goodwin, K.S. Sin. "Adaptive filtering Prediction and Control". Dover Publications, New York, 1984. ISBN: 9780486137728.

[9] A. Vahidi, A. Stefanopoulou, H. Peng. "Recursive least squares with forgetting for online estimation of vehicle mass and road grade: Theory and experiments". Vehicle System Dynamics, 2005: 43, pp.31-55.

[10] M. Guay. "A time-varying extremum-seeking control approach for discrete-time systems". Journal of Process Control 24, 2014, pp. 98-112. 
[11] J.F. Forbes, T.E. Marlin. "Model Accuracy for Economic Optimizing Controllers: The Bias Update Case". Industrial \& Engineering Chemistry Research, 1994; 33, pp.1919-1929.

[12] Y. Zhang, J.F. Forbes. "Extended design cost: a performance criterion for real-time optimization systems". Computers \& Chemical Engineering, 2000; 24, pp.1829-1841. 